\title{
Positive Predictive Value of Diagnostic Test
}

National Cancer Institute

\section{Source}

National Cancer Institute. Positive Predictive Value of Diagnostic Test. NCI Thesaurus.

Code C41389.

The probability that an individual is affected with the condition when a positive test result is observed. Predictive values should only be calculated from cohort studies or studies that legitimately reflect the number of people in the population who have the condition of interest at that time since predictive values are inherently dependent upon the prevalence. PPVDT can be determined by calculating: number of true positive results divided by the sum of true positive results plus number of false positive results. 